\title{
PCSP 1.0 Software for Partial Credit S-P Chart Analysis
}

\author{
Duchieu Pham, Tianwei Sheu, and Masatake Nagai \\ Graduate Institute of Educational Information and Measurement, \\ National Taichung University of Education, Taichung, Taiwan \\ hieuhagdthsp2@gmail.com
}

\begin{abstract}
S-P Chart Analysis is an effective method to diagnose the learning status of students and items quality. This method is very suitable for use in adaptive learning systems. However, it only handles dichotomous data without considering the test data of partial credit scoring. The main purpose of this study is to develop a tool for analyzing the test data of partial credit scoring according to S-P Chart Analysis method. First, the study proposed the Partial Credit S-P Chart Analysis. Then, the study developed the software to analyze partial credit data according to the method proposed. PCSP 1.0 software developed by this study can help the user to reduce the complex mathematical calculations and provide results accurately and quickly. The interaction between users and the software is very easy through a graphical user interface. PCSP 1.0 software handles not only dichotomous data but also partial credit data. This software is an actually useful tool for evaluating and diagnosing the achievement of students.
\end{abstract}

Keywords: Dichotomous, learning status, S-P Chart, partial credit scoring.

\section{Introduction}

Student-Problem (S-P) Chart Analysis was invented in 1970 by Takahiro Sato. It is an effective method to diagnose the learning status of students and item quality. Its purpose is to help teachers diagnose the abnormal response of students as well as items [1, 2]. S-P Chart Analysis analyzes the student's response patterns to items in a test. Based on a number of indexes including Disparity Coefficient, Homogeneity Coefficient, Caution Index for Student and Caution Index for Problem, S-P Chart Analysis evaluates whether a response pattern is abnormal [3]. As a method of nonparametric statistics, S-P Chart Analysis doesn't require any assumptions to the characteristics of population. This test data analysis method is very suitable for analyzing small classes, and is particularly useful in formative assessment [4]. It helps teachers analyze test items, diagnose the learning difficulties of students, identify the types of learning and understand the individual differences in students' learning. Thereby, teachers can have the appropriate choice of teaching content, teaching plan and teaching method in order to improve the academic achievement of students [5].

S-P Chart Analysis has been widely used in many educational researches [6-9]. At the same time, many educational researchers have combined S-P Chart Analysis method with different approaches to form new effective methods in educational measurement and evaluation [10-13]. However, S-P Chart Analysis has two shortcomings. The first one is that both the Caution Index for Student and the Caution Index for Problem are not normalized. Thus, the Caution Indexes of two different students are not comparable. Harnisch and Linn [14] developed the Modified Caution Index (MCI) which normalizes Sato's Caution Index to solve the first problem. The second problem is this method only analyzes dichotomous data 
without considering the test data of partial credit scoring. When test data is not dichotomous, before using Sato's S-P Chart Analysis, this data must be converted to dichotomous data. The common conversion way uses a threshold value. If the score which a student achieves on an item is larger than the threshold, it is converted to 1 . Conversely, the score is converted to 0. Two disadvantages of this way are: First, the diagnosis result depends on the selected threshold; Second, useful information in partial score is removed.

This study proposed an extension of S-P Chart Analysis for analyzing the test data of partial credit scoring. This new proposal can simultaneously handle two shortcomings mentioned above. The study process includes two basic steps. In step 1, this study proposed the Partial Credit S-P Chart Analysis based on the method of Harnisch and Linn [14]. The main points of new proposal are to extend the mathematical formulas of the Student's Modified Caution Index and the Problem's Modified Caution Index. Unlike Sato's S-P Chart Analysis, Partial Credit S-P Chart Analysis considers the test whose question is scored from 0 to 1 . In step 2, the study developed PCSP 1.0 software to analyze partial credit data according to the method proposed. The software is developed by the MATLAB programming language that has powerful performance in numerical computation, symbolic operation and graphical representation [15-18]. PCSP 1.0 software handles not only dichotomous data but also partial credit data. It helps the user to reduce the complex mathematical calculations and provide results accurately and quickly. In addition, the interaction between users and the software is very easy through a graphical user interface. The software can completely integrate into adaptive learning systems to improve teaching effectiveness.

\section{S-P Chart Analysis}

The basic theory of S-P Chart Analysis is summarized in the following description $[5,14,19-21]$ :

\subsection{Dichotomous Response Matrix of S-P Chart}

\section{Definition 1: Dichotomous Response Matrix}

Suppose that $m$ students take a test of $n$ problems. Let $Y$ denote the dichotomous response matrix.

$Y=\left[y_{i j}\right]_{m \times n}$

where, $i=1,2, \cdots, m ; j=1,2, \cdots, n$;

$y_{i j}=1$ if the $i^{t h}$ student answers the $j^{\text {th }}$ problem correctly, $y_{i j}=0$ if the $i^{\text {th }}$ student answers the $j^{\text {th }}$ problem incorrectly.

\section{Definition 2: Dichotomous Response Matrix of S-P Chart}

The dichotomous response matrix $Y=\left[y_{i j}\right]_{m \times n}$ is called the dichotomous response matrix of S-P Chart when both of two following conditions are satisfied:

$+y_{1} \geq y_{2} \geq \cdots \geq y_{m}$, where, $y_{i \bullet}=\sum_{j=1}^{n} y_{i j}$.
$+y_{\bullet 1} \geq y_{\bullet 2} \geq \cdots \geq y_{\bullet}$, where, $y_{\bullet}=\sum_{i=1}^{m} y_{i j}$. 


\subsection{Student Curve and Problem Curve}

After the dichotomous response matrix of S-P Chart is formed, the student curve and the problem curve are defined as follows:

Based on the total score of each student, the number of problems corresponding to the total score is counted from left to right. Then, the dividing line is drawn on the right of these problems. Lastly, all the dividing lines are connected by line segments. The staircase curve which is formed according to the way above is called student curve (S-curve).

Based on the number of correct answers of each problem, the number of students corresponding to the number of correct answers is counted from top to bottom. Then, the dividing line is drawn on the bottom of these students. Lastly, all the dividing lines are connected by line segments. The staircase curve which is formed according to the way above is called problem curve ( $\mathrm{P}$-curve).

\subsection{The Disparity Coefficient of S-P Chart}

\section{Definition 3: Disparity Coefficient}

Suppose that $Y=\left[y_{i j}\right]_{m \times n}$ is the dichotomous response matrix of S-P Chart. Let $D *$ be the Disparity Coefficient.

$$
D^{*}=\frac{S(m, n, \bar{P})}{S_{B}(m, n, \bar{P})}
$$

where, $\bar{P}=\frac{1}{m n} \sum_{i=1}^{m} y_{i \bullet}=\frac{1}{m n} \sum_{j=1}^{n} y_{\bullet}$.

$S(m, n, \bar{P})$ is the area bounded by the S-curve and the P-curve in the real S-P chart.

$S_{B}(m, n, \bar{P})$ is the area bounded by the S-curve and the P-curve if both of two curves are the cumulative distribution function curves of binomial distribution.

In S-P Chart Analysis, the Disparity Coefficient is the index that indicates the non-homogeneity degree of the test data. The value of the Disparity Coefficient is between 0 and 1. In norm-referenced test, the standard value of $D *$ is 0.5 . In criterion-referenced test, the standard value of $D^{*}$ is 0.4 . If $D^{*}>0.6$ for normreferenced test or $D^{*}>0.5$ for criterion-referenced test, then the test contains a lot of heterogeneous factors.

\subsection{Modified Caution Index of S-P Chart}

In S-P Chart Analysis method of Sato, Caution Index displays the abnormal level of the response pattern. There are two types of Caution Index. The first type is the Caution Index for Student and the second one is the Caution Index for Problem. The Sato's Caution Index is not normalized. So, Harnisch and Linn [14] have normalized the Sato's Caution Index and proposed the Modified Caution Index. The formulas of Modified Caution Indexes are as follows:

+ The Modified Caution Index of the $i^{\text {th }}$ student:

$$
\text { MCI Si }=\frac{\sum_{j=1}^{y_{i}}\left(1-y_{i j}\right)\left(y_{\bullet}\right)-\sum_{j=y_{i \bullet}+1}^{n}\left(y_{i j}\right)\left(y_{\bullet}\right)}{\sum_{j=1}^{y_{i}}\left(y_{\bullet}\right)-\sum_{j=n+1-y_{\bullet}}^{n}\left(y_{\bullet}\right)}
$$

+ The Modified Caution Index of the $j^{\text {th }}$ problem: 


$$
M C I \quad P_{P j}=\frac{\sum_{i=1}^{y \cdot j}\left(1-y_{i j}\right)\left(y_{i \bullet}\right)-\sum_{i=y \cdot j+1}^{m}\left(y_{i j}\right)\left(y_{\bullet \bullet}\right)}{\sum_{i=1}^{y \cdot j}\left(y_{i \bullet}\right)-\sum_{i=m+1-y \cdot j}^{m}\left(y_{\bullet}\right)}
$$

where, $y_{i \bullet}=\sum_{j=1}^{n} y_{i j}$ and $y_{\bullet} \cdot=\sum_{i=1}^{m} y_{i j}$.

If $\mathrm{MCI}$ is greater than 0.3 , it suggests that there are some abnormalities in the response pattern. If MCI is greater than 0.5, the anomaly of the response pattern is serious. Table 1 describes S-P Chart with Modified Caution Index.

Table 1. S-P Chart with Modified Caution Index

\begin{tabular}{|c|c|c|c|c|}
\hline & $\begin{array}{c}\text { Problem number } \\
P_{j}, j=1,2, \cdots, n\end{array}$ & Total score & Ratio & MCI \\
\hline $\begin{array}{c}\text { Student number } \\
S_{i}, i=1,2, \cdots, m\end{array}$ & $Y=\left[y_{i j}\right]_{m \times n}$ & $\begin{array}{c}\text { High } \\
\text { Low }\end{array}$ & $\frac{S S_{i}}{n}$ & MCI $S_{i}$ \\
\hline $\begin{array}{c}\text { Number of } \\
\text { correct answer }\end{array}$ & $\begin{array}{c}\text { More } \leftrightarrow \text { Less } \\
P P_{j}\end{array}$ & $\sum_{i=1}^{m} S S_{i}=\sum_{j=1}^{n} P P_{j}$ & & \\
\hline Ratio & $\frac{P P_{j}}{m}$ & & & \\
\hline MCI & $M C I P_{j}$ & & & \\
\hline
\end{tabular}

Note. Adapted from "Analysis of item response patterns: Questionable test data and dissimilar curriculum practices" by D. L. Harnisch and R. L. Linn, Journal of Educational Measurement, vol. 18, no 3, (1981), pp 133-146.

\section{The New Proposal for Partial Credit S-P Chart}

In Partial Credit S-P Chart, if a student completes the question, his score is 1 . If the student only completes a part of the question, his score is corresponding to the completed part. This study redefined the MCI of Harnisch and Linn [14]. Based on the definition, the study extends the formulas of the Student's MCI and the Problem's MCI for partial credit data. The definition of MCI and the formulas proposed by this paper are as follows:

\section{Definition 4: Modified Caution Index}

Let $M C I$ be the Modified Caution Index. $M C I$ is defined by the following formula:

$$
M C I=\frac{\operatorname{cov}\left(X^{*}, p\right)-\operatorname{cov}(X, p)}{\operatorname{cov}\left(X^{*}, p\right)-\operatorname{cov}\left(X^{\prime}, p\right)}
$$

where, $p$ is the vector of the correct response rates, $X$ is the vector of the real response pattern, $X *$ is the vector of the perfect response pattern, $X^{\prime}$ is the vector of the perfectly-inconsistent response pattern.

\section{Theorem 1: Student's Modified Caution Index for Partial Credit S-P Chart}

Suppose the response matrix of Partial Credit S-P Chart is $Y=\left[y_{i j}\right]_{m \times n}$. The student's Modified Caution Index 


$$
M C I S i=\frac{\sum_{j=1}^{l}\left(1-y_{i j}\right)\left(y_{\bullet}\right)+\left(y_{i}-l\right)\left(y_{\bullet}(l+1)\right)-\sum_{j=l+1}^{n}\left(y_{i j}\right)\left(y_{\bullet}\right)}{\sum_{j=1}^{l}\left(y_{\bullet}\right)+\left(y_{i}-l\right)\left(y_{\bullet}(l+1)-y_{\bullet}(n-l)\right)-\sum_{j=n-l+1}^{n}\left(y_{\bullet}\right)}
$$

where, $y_{i \bullet}=\sum_{j=1}^{n} y_{i j}$ and $y_{\bullet} j_{j}=\sum_{i=1}^{m} y_{i j}, l=\left[y_{i \bullet}\right]$ is the largest integer not greater than $y_{i \bullet}$.

\section{Proof.}

Suppose that $Y=\left[y_{i j}\right]_{m \times n}$ is the response matrix of Partial Credit S-P Chart. Then the vector of the problems' correct response rates is:

$$
p=\left(p_{p_{1}}, p_{p_{2}}, \cdots, p_{p_{j}}, \cdots, p_{p_{n}}\right) \text {, where, } p_{p_{j}}=\frac{y_{\bullet} j}{m}, y_{\bullet}=\sum_{i=1}^{m} y_{i j} .
$$

Consider the $i^{\text {th }}$ student. Then, the vector of the real response pattern, the vector of the perfect response pattern and the vector of the perfectly-inconsistent response pattern are as follows:

+ The vector of the real response pattern:

$$
X=\left(y_{i 1}, y_{i 2}, \cdots, y_{i j}, \cdots, y_{i n}\right)
$$

+ The vector of the perfect response pattern:

$$
X^{*}=\left(X_{1}^{*}, X_{2}^{*}, \cdots, X_{j}^{*}, \cdots, X_{n}^{*}\right), \text { where, } X_{j}^{*}= \begin{cases}1 & \text { if } \mathrm{j} \leq\left[y_{i \bullet}\right] \\ y_{i \bullet}-\left[y_{i \bullet}\right] & \text { if } \mathrm{j}=\left(\left[y_{i \bullet}\right]+1\right) \\ 0 & \text { if } \mathrm{j}>\left(\left[y_{i \bullet}\right]+1\right)\end{cases}
$$

+ The vector of the perfectly-inconsistent response pattern:

$$
X^{\prime}=\left(X_{1}^{\prime}, X_{2}^{\prime}, \cdots, X_{j}^{\prime}, \cdots, X_{n}^{\prime}\right), \text { where, } X_{j}^{\prime}= \begin{cases}0 & \text { if } \mathrm{j} \leq\left(n-\left[y_{i \bullet}\right]-1\right) \\ y_{i \bullet}-\left[y_{i \bullet}\right] & \text { if } \mathrm{j}=\left(n-\left[y_{i \bullet}\right]\right) \\ 1 & \text { if } \mathrm{j}>\left(n-\left[y_{i \bullet}\right]\right)\end{cases}
$$

The formula for the covariance:

$$
\operatorname{cov}(x, y)=E[x . y]-E[x] \cdot E[y]
$$

So,

$$
\begin{aligned}
& \operatorname{cov}(X, p)=\frac{1}{n} \sum_{j=1}^{n}\left(y_{i j}\right)\left(\frac{y_{\bullet} j}{m}\right)-\frac{y_{i \bullet}}{n}\left(\frac{1}{n} \sum_{j=1}^{n} \frac{y_{\bullet} j}{m}\right) \\
& \operatorname{cov}\left(X^{*}, p\right)=\frac{1}{n}\left(\sum_{j=1}^{l} \frac{y_{\bullet}}{m}+\left(y_{i \bullet}-l\right) \frac{y_{\bullet}(l+1)}{m}\right)-\frac{y_{i \bullet}}{n}\left(\frac{1}{n} \sum_{j=1}^{n} \frac{y_{\bullet}}{m}\right), \text { where, } l=\left[y_{i \bullet}\right] . \\
& \operatorname{cov}\left(X^{\prime}, p\right)=\frac{1}{n}\left(\left(y_{i \bullet}-l\right) \frac{y_{\bullet}(n-l)}{m}+\sum_{j=n-l+1}^{n} \frac{y_{\bullet} j}{m}\right)-\frac{y_{i \bullet}}{n}\left(\frac{1}{n} \sum_{j=1}^{n} \frac{y_{\bullet} j}{m}\right)
\end{aligned}
$$

Thus,

$$
\operatorname{cov}\left(X^{*}, p\right)-\operatorname{cov}(X, p)=\frac{1}{n m}\left(\sum_{j=1}^{l} y_{\bullet}+\left(y_{i \bullet}-l\right) y_{\bullet}(l+1)-\sum_{j=1}^{n}\left(y_{i j}\right)\left(y_{\bullet}\right)\right)
$$

and:

$$
\operatorname{cov}\left(X^{*}, p\right)-\operatorname{cov}\left(X^{\prime}, p\right)=\frac{1}{n m}\left(\sum_{j=1}^{l} y_{\bullet_{j}}+\left(y_{i \bullet}-l\right)\left(y_{\bullet}(l+1)-y_{\bullet}(n-l)\right)-\sum_{j=n-l+1}^{n} y_{\bullet}\right)
$$

Substituting (I) and (II) into (9). The Modified Caution Index of the $i^{t h}$ student is:

$$
M C I S i=\frac{\sum_{j=1}^{l}\left(1-y_{i j}\right)\left(y_{\bullet}\right)+\left(y_{i \bullet}-l\right)\left(y_{\bullet}(l+1)\right)-\sum_{j=l+1}^{n}\left(y_{i j}\right)\left(y_{\bullet}\right)}{\sum_{j=1}^{l}\left(y_{\bullet}\right)+\left(y_{i \bullet}-l\right)\left(y_{\bullet}(l+1)-y_{\bullet}(n-l)\right)-\sum_{j=n-l+1}^{n}\left(y_{\bullet}\right)} \text { (Q.E.D). }
$$




\section{Theorem 2: Problem's Modified Caution Index for Partial Credit S-P Chart}

Suppose that the response matrix of Partial Credit S-P Chart is $Y=\left[y_{i j}\right]_{m \times n}$. The problem's Modified Caution Index

$$
M C I \quad P_{P i}=\frac{\sum_{i=1}^{l^{\prime}}\left(1-y_{i j}\right)\left(y_{i \bullet}\right)+\left(y_{\bullet}-l^{\prime}\right)\left(y_{\left(l^{\prime}+1\right) \bullet}\right)-\sum_{i=l^{\prime}+1}^{m}\left(y_{i j}\right)\left(y_{i \bullet}\right)}{\sum_{i=1}^{l^{\prime}}\left(y_{\boldsymbol{\bullet}}\right)+\left(y_{\bullet}-l^{\prime}\right)\left(y_{\left(l^{\prime}+1\right) \bullet}-y_{\left(m-l^{\prime}\right)}\right)-\sum_{i=m-l^{\prime}+1}^{m}\left(y_{i \bullet}\right)}
$$

where, $y_{i \bullet}=\sum_{j=1}^{n} y_{i j}$ and $y_{\bullet} j=\sum_{i=1}^{m} y_{i j}, l^{\prime}=\left[y_{\bullet}\right]$ is the largest integer not greater than $y_{\bullet_{j}}$.

Proof.

The proof of theorem 2 is similar to the one of theorem 1 . Therefore, this paper does not present it.

\section{Software Development}

\subsection{Process for Software Development}

The progress for PCSP 1.0 software development is the 6-step process described in Figure 1.

\subsection{The Algorithms of the Program}

The main algorithm of the program is described in Figure 2. The procedure for calculating MCI is the most important part of the program. Its algorithm and the algorithm for diagnosing student type are described in this section.

\subsubsection{Algorithm for Calculating Modified Caution Index}

procedure CALCULATE_MODIFIED_CAUTION_INDEX

input $Y=\left[y_{i j}\right]_{m \times n} ; \% Y$ is the response matrix of Partial Credit S-P Chart.

$y_{S}=\left(y_{1}, y_{2}, \cdots, y_{i \bullet}, \cdots, y_{m} \bullet\right) ; \% y_{S}$ is the vector that shows the total score of each student.

$$
\% y_{i \bullet}=\sum_{j=1}^{n} y_{i j} .
$$

$y_{P}=\left(y_{\bullet_{1}}, y_{\bullet}, \cdots, y_{\bullet}, \cdots, y_{\bullet}\right) ; \% y_{P}$ is the vector that shows the correct total for each item.

$$
\% y_{\bullet}{ }_{j}=\sum_{i=1}^{m} y_{i j} .
$$

for $i=1$ to $m$ do

Calculating $M C I S i$ according to (8).

\section{end do}

for $j=1$ to $n$ do

Calculating $\mathrm{MCI}_{P_{j}}$ according to (9).

end do

output $\mathrm{MCI}_{s} ; \% \mathrm{MCI}_{s}$ is the vector of the student's MCI.

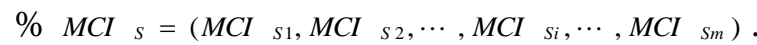

output $\mathrm{MCI}_{P} ; \% \mathrm{MCI}_{P}$ is the vector of the problem's MCI.

return

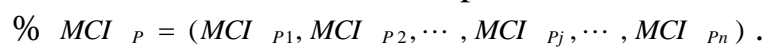




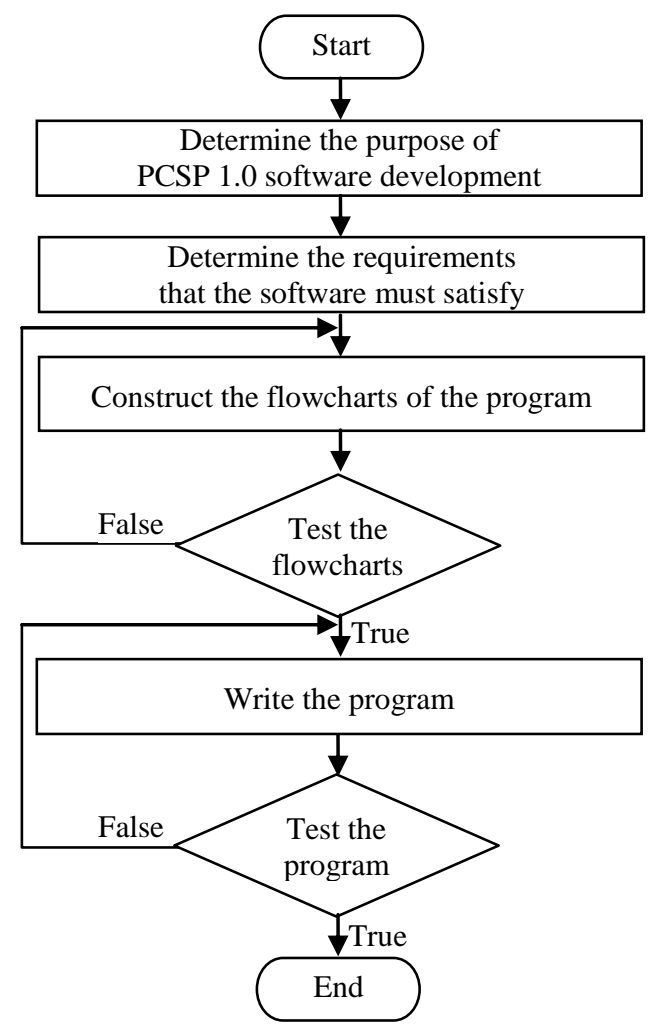

Figure 1. Development Process

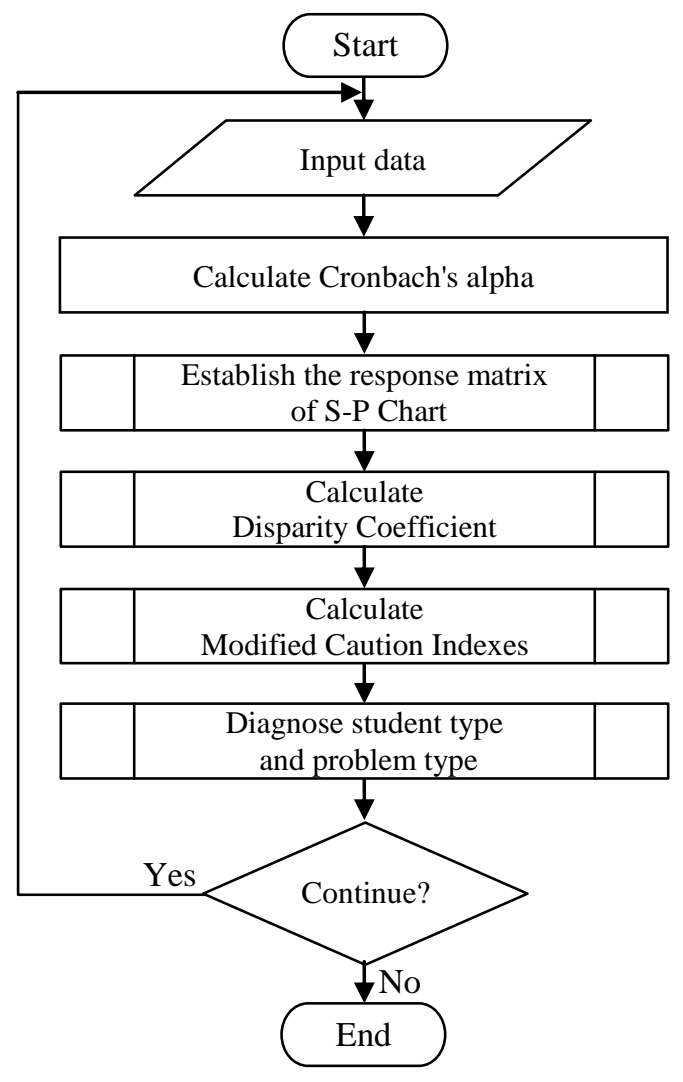

Figure 2. Main algorithm

\subsubsection{Algorithm for Diagnosing Student Type}

\section{procedure DIAGNOSE_STUDENT_TYPE}

input $y_{S} ; \% y_{S}$ is the vector that shows the total score of each student.

$$
\% y_{S}=\left(y_{1}, y_{2}, \cdots, y_{i_{\bullet}}, \cdots, y_{m} \bullet\right) \text {. }
$$

input $M C I_{s} ; \%$ MCI $_{s}$ is the vector of the student's MCI.

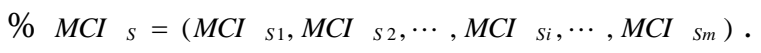

$$
\text { for } i=1 \text { to } m \text { do }
$$$$
p_{S_{i}}=\frac{y_{i \bullet}}{n} ;
$$

if $p_{S_{i}}>0.5$ and $M C I s_{i} \leq 0.3$ then

The $i^{\text {th }}$ student belongs to A type.

elseif $p_{S_{i}}>0.5$ and $M C I S_{S_{i}}>0.3$ then

The $i^{\text {th }}$ student belongs to B type.

elseif $p_{S_{i}} \leq 0.5$ and $M C I s_{i} \leq 0.3$ then

The $i^{\text {th }}$ student belongs to $\mathrm{C}$ type.

$$
\text { else }
$$

The $i^{\text {th }}$ student belongs to D type.

\section{end if}

end do

return 


\subsection{Working with PCSP 1.0 Software}

\subsubsection{Software Requirements Specification}

1) Windows $X P$, Windows 7 or upgrade versions.

2) Screen resolution $1024 \times 768$.

3) MATLAB version 7.10 or upgrade versions.

\subsubsection{Graphical User Interface}

The user interacts with PCSP 1.0 software through the graphical user interface (GUI). The operations are easily performed by selecting the corresponding menu on the menu bar. The role of each menu is described in detail in Table 2. Data in a selected folder is imported through submenu "Input data" of FILE menu. Depending on the choice of the user on ANALYSIS menu, the software can provide them with results such as Cronbach's alpha, S-P chart, the S-P curve graph, the diagnosis graph for students, the diagnosis graph for problems, the distribution graph of total score and the distribution graph of problem difficulty.

Table 2. The Function of the Menus

\begin{tabular}{|l|l|l|}
\hline \multicolumn{1}{|c|}{ Menu } & \multicolumn{1}{|c|}{ Menu item } & \multicolumn{1}{c|}{ Function } \\
\hline \multirow{4}{*}{ I. File } & 1. Input data & Input data. \\
\cline { 2 - 3 } & 2. Quit & Quit the software. \\
\hline \multirow{4}{*}{ II. Analysis } & 1. S-P chart & Calculate and output the results of S-P chart. \\
\cline { 2 - 3 } & 2. S-P curve & Draw the S-P curve graph. \\
\cline { 2 - 3 } & 3. Diagnosis graph & $\begin{array}{l}\text { Present the diagnosis graph for students and the } \\
\text { diagnosis graph for problems. }\end{array}$ \\
\cline { 2 - 3 } & 4. Distribution & $\begin{array}{l}\text { Display the distribution graph of total score and the } \\
\text { distribution graph of problem difficulty. }\end{array}$ \\
\hline
\end{tabular}

\section{Application Example}

This study uses the test data consisting of the response patterns of 27 secondary school students to illustrate the application of PCSP 1.0 software. These students participate in a partial credit test of 10 items. The test data are inputted into PCSP 1.0 software. Figure 3 shows the S-P chart for the test data that are used in this study and Figure 4 describes the S-P curve graph.

After analyzing data, PCSP 1.0 software displays the most important analysis results in S-P chart. S-P chart contains the information about total score of students, correct answer rate of students, the students' Modified Caution Index, student types, correct answer total of problems, correct answer rate of problems, the problems' Modified Caution Index and problem types. PCSP 1.0 software also provides the results of student diagnosis and problem diagnosis by diagnostic graphs as Figure 5 and Figure 6. In the diagnostic graph, students are divided into four types A, B, C and D. The similar thing is also done for questions. The characteristics of each type are presented in Table 3. In addition, PCSP 1.0 software also displays the distribution graph of total score and the distribution graph of problem difficulty in two types of statistics graph: boxplot and histogram. 


\begin{tabular}{|c|c|c|c|c|c|c|c|c|c|c|c|c|c|c|c|}
\hline S-P & P2 & P10 & P3 & P8 & P1 & P9 & P4 & P5 & P7 & P6 & Total & Ratio & $\mathrm{MCl}$ & Type & \multirow{10}{*}{\begin{tabular}{|c|} 
Students \\
27
\end{tabular}} \\
\hline S07 & 1 & 1 & 1 & 1 & 1 & 1 & 1 & 0.9 & 0.7 & 0 & 8.6 & 0.86 & 0.01 & A & \\
\hline S15 & 1 & 0.4 & 1 & 1 & 1 & 1 & 1 & 0.4 & 0.9 & 0 & 7.7 & 0.77 & 0.25 & A & \\
\hline S06 & 0.6 & 1 & 1 & 1 & 0.7 & 1 & 1 & 0.2 & 0.2 & 1 & 7.7 & 0.77 & 0.41 & $B$ & \\
\hline S11 & 1 & 1 & 1 & 1 & 0.3 & 1 & 1 & 0.4 & 0.9 & 0 & 7.6 & 0.76 & 0.22 & A & \\
\hline S17 & 1 & 1 & 1 & 0.5 & 0.7 & 1 & 0 & 1 & 0.2 & 0.8 & 7.2 & 0.72 & 0.33 & B & \\
\hline S14 & 0.6 & 1 & 1 & 1 & 0.7 & 1 & 0.5 & 0.4 & 0.2 & 0.2 & 6.6 & 0.66 & 0.21 & A & \\
\hline S27 & 0.7 & 1 & 0.8 & 1 & 0.7 & 1 & 0 & 0.7 & 0.6 & 0 & 6.5 & 0.65 & 0.22 & A & \\
\hline S24 & 0.7 & 0.6 & 1 & 1 & 0.7 & 1 & 0.5 & 0.4 & 0.4 & 0 & 6.3 & 0.63 & 0.24 & A & \\
\hline $\mathbf{S} 16$ & 0.7 & 1 & 1 & 0.8 & 0.7 & 1 & 0.2 & 0.4 & 0 & 0 & 5.8 & 0.58 & 0.13 & A & \\
\hline $\mathrm{S} 22$ & 0.7 & 0 & 0.8 & 1 & 1 & 0 & 0 & 0.7 & 0.4 & 1 & 5.6 & 0.56 & 0.56 & $B$ & \multirow{2}{*}{ Problems } \\
\hline $\mathrm{S} 20$ & 0.6 & 1 & 1 & 1 & 0.4 & 1 & 0 & 0.6 & 0 & 0 & 5.6 & 0.56 & 0.14 & A & \\
\hline S01 & 0.9 & 1 & 0 & 1 & 0.6 & 0 & 1 & 0 & 0.7 & 0 & 5.2 & 0.52 & 0.31 & B & \multirow{8}{*}{$\begin{array}{c}\text { Cronbach Alpha } \\
\qquad 0.87398\end{array}$} \\
\hline$S 13$ & 0.8 & 0 & 1 & 0.6 & 0.6 & 1 & 0 & 0.5 & 0 & 0 & 4.5 & 0.45 & 0.18 & C & \\
\hline S02 & 0.2 & 1 & 0.5 & 0 & 0.7 & 1 & 0.4 & 0.2 & 0 & 0 & 4 & 0.40 & 0.23 & C & \\
\hline$S 10$ & 0.2 & 0.5 & 1 & 0 & 1 & 0 & 1 & 0.3 & 0 & 0 & 4 & 0.40 & 0.33 & D & \\
\hline S03 & 1 & 0 & 0.4 & 0.4 & 0.3 & 0.3 & 0.4 & 0.2 & 0 & 0.6 & 3.6 & 0.36 & 0.36 & $D$ & \\
\hline S05 & 0.5 & 1 & 0 & 0 & 0.3 & 0 & 0.4 & 0 & 0 & 0 & 2.2 & 0.22 & 0.17 & C & \\
\hline S04 & 0.3 & 1 & 0 & 0.4 & 0 & 0 & 0 & 0 & 0.2 & 0 & 1.9 & 0.19 & 0.17 & C & \\
\hline$S 19$ & 1 & 0.2 & 0 & 0 & 0.7 & 0 & 0 & 0 & 0 & 0 & 1.9 & 0.19 & 0.06 & $c$ & \\
\hline S26 & 1 & 0 & 0 & 0 & 0.3 & 0 & 0 & 0.3 & 0 & 0 & 1.6 & 0.16 & 0.14 & $C$ & \multirow{2}{*}{ Disparity index } \\
\hline 509 & 0.2 & 1 & 0 & 0 & 0 & 0 & 0 & 0 & 0 & 0 & 1.2 & 0.12 & 0.11 & C & \\
\hline $\mathrm{S} 12$ & 0.8 & 0 & 0 & 0 & 0 & 0 & 0 & 0.3 & 0 & 0 & 1.1 & 0.11 & 0.17 & c & \multirow[t]{10}{*}{0.45} \\
\hline S18 & 0 & 0 & 0 & 0.8 & 0 & 0 & 0 & 0 & 0.2 & 0 & 1 & 0.10 & 0.36 & D & \\
\hline S25 & 0.7 & 0 & 0 & 0 & 0 & 0 & 0 & 0 & 0.3 & 0 & 1 & 0.10 & 0.25 & C & \\
\hline S21 & 0.3 & 0 & 0 & 0 & 0.2 & 0 & 0 & 0 & 0 & 0 & 0.5 & 0.05 & 0.12 & C & \\
\hline S08 & 0.2 & 0 & 0 & 0 & 0 & 0 & 0 & 0 & 0 & 0 & 0.2 & 0.02 & 0.00 & C & \\
\hline S23 & 0 & 0 & 0 & 0 & 0.2 & 0 & 0 & 0 & 0 & 0 & 0.2 & 0.02 & 0.30 & C & \\
\hline Total & 16.7 & 14.7 & 13.5 & 13.5 & 12.8 & 12.3 & 8.4 & 7.9 & 5.9 & 3.6 & 109.3 & & & & \\
\hline Ratio & 0.62 & 0.54 & 0.50 & 0.50 & 0.47 & 0.46 & 0.31 & 0.29 & 0.22 & 0.13 & & & & & \\
\hline $\mathrm{MCl}$ & 0.29 & 0.19 & 0.03 & 0.09 & 0.20 & 0.04 & 0.15 & 0.19 & 0.18 & 0.22 & & & & & \\
\hline Type & A & A & A & C & C & C & C & C & C & C & & & & & \\
\hline
\end{tabular}

Figure 3. The result of Partial Credit S-P Chart

Table 3. Student Types and Problem Types

\begin{tabular}{|c|l|l|}
\hline Type & \multicolumn{1}{|c|}{ Student } & \multicolumn{1}{c|}{ Problem } \\
\hline A & Good learning, high stability. & $\begin{array}{l}\text { Good problem, suitable for distinguishing } \\
\text { between low achievers and the others. }\end{array}$ \\
\hline B & $\begin{array}{l}\text { Learning performance is high but } \\
\text { stability is low; Carelessness. }\end{array}$ & $\begin{array}{l}\text { Problem has anomalous parts, need to be } \\
\text { partially amended. }\end{array}$ \\
\hline C & $\begin{array}{l}\text { Lack of scholastic ability, } \\
\text { insufficient learning, need to more } \\
\text { effort. }\end{array}$ & $\begin{array}{l}\text { Difficult problem, suitable for distinguishing } \\
\text { between high achievers and the others. }\end{array}$ \\
\hline D & $\begin{array}{l}\text { Weak learning, lack of } \\
\text { preparation, low stability. }\end{array}$ & $\begin{array}{l}\text { Poor problem, the data are incorrect or the } \\
\text { meaning is ambiguous, need to be repaired. }\end{array}$ \\
\hline
\end{tabular}




\begin{tabular}{|c|c|c|c|c|c|c|c|c|c|c|c|}
\hline & P2 & P10 & P3 & P8 & P1 & P9 & P4 & P5 & P7 & P6 & \\
\hline S07 & 1 & 1 & 1 & 1 & 1 & 1 & 1 & 0.9 & 0.1 & 0 & \\
\hline$s 15$ & 1 & 0.4 & 1 & 1 & 1 & 1 & 1 & 0.4 & 0.9 & 0 & \\
\hline so6 & 0.6 & 1 & 1 & 1 & 0.7 & 1 & 1 & 0.2 & 0.2 & 1 & \\
\hline$s 11$ & 1 & 1 & 1 & 1 & 0.3 & 1 & 1 & 0.5 & 0.9 & $=-0=-\square$ & \\
\hline $\mathbf{s} 17$ & 1 & 1 & 1 & 0.5 & 0.7 & 1 & 0 & 1 & 0.2 & 0.8 & \\
\hline $\mathbf{s} 14$ & 0.6 & 1 & 1 & 1 & 0.7 & 1 & 0.5 & 0.4 & 02 & 0.2 & \\
\hline S27 & 0.7 & 1 & 0.8 & 1 & 0.7 & 1 & $\delta$ & 0.7 & 0.6 & 0 & \\
\hline s24 & 0.7 & 0.6 & 1 & 1 & 0.7 & 1 & $\sqrt{0.5}$ & 0.4 & 0.4 & 0 & \\
\hline$s 16$ & 0.7 & 1 & 1 & 0.8 & 0.7 & 1 & $\theta .2=$ & 0.4 & 0 & 0 & \\
\hline s22 & 0.7 & 0 & 0.8 & 1 & 1 & of & 0 & 0.7 & 0.4 & 1 & \\
\hline$s 20$ & 0.6 & 1 & 1 & 1 & 0.4 & 1 & 0 & 0.6 & 0 & 0 & \\
\hline so1 & 0.9 & 1 & 0 & 1 & 0.6 & 0 & 1 & 0 & 0.7 & 0 & \\
\hline $\mathbf{s} 13$ & 0.8 & 0 & 1 & 0.6 & $0 \sqrt{6}$ & $-\tau^{-}$ & 0 & 0.5 & 0 & 0 & S Soune \\
\hline so2 & 0.2 & 1 & $1=-0.5-$ & $\theta=-$ & 0.7 & 1 & 0.4 & 0.2 & 0 & 0 & - surve \\
\hline$s 10$ & 0.2 & $--0.5=$ & 1 & 0 & 1 & 0 & 1 & 0.3 & 0 & 0 & $=-P$ curve \\
\hline SO3 & 1 & 0 & 0.4 & 0.4 & 0.3 & 0.3 & 0.4 & 0.2 & 0 & 0.6 & \\
\hline SO5 & I & 1 & 0 & 0 & 0.3 & 0 & 0.4 & 0 & 0 & 0 & \\
\hline SO4 & 0.3 & 1 & 0 & 0.4 & 0 & 0 & 0 & 0 & 0.2 & 0 & \\
\hline$s 19$ & 1 & 0.2 & 0 & 0 & 0.7 & 0 & 0 & 0 & 0 & 0 & \\
\hline $\mathbf{s} 26$ & 1 & 0 & 0 & 0 & 0.3 & 0 & 0 & 0.3 & 0 & 0 & \\
\hline sog & 0.2 & 1 & 0 & 0 & 0 & 0 & 0 & 0 & 0 & 0 & \\
\hline $\mathbf{s} 12$ & 0.8 & 0 & 0 & 0 & 0 & 0 & 0 & 0.3 & 0 & 0 & \\
\hline $\mathbf{s} 18$ & 0 & 0 & 0 & 0.8 & 0 & 0 & 0 & 0 & 0.2 & 0 & \\
\hline $\mathbf{s} 25$ & 0.7 & 0 & 0 & 0 & 0 & 0 & 0 & 0 & 0.3 & 0 & \\
\hline $\mathbf{s} 21$ & $0 \longdiv { 3 }$ & 0 & 0 & 0 & 0.2 & 0 & 0 & 0 & 0 & 0 & \\
\hline S08 & 0.2 & 0 & 0 & 0 & 0 & 0 & 0 & 0 & 0 & 0 & \\
\hline$\$ 23$ & 0 & 0 & 0 & 0 & 0.2 & 0 & 0 & 0 & 0 & 0 & \\
\hline
\end{tabular}

Figure 4. The Student Curve and the Problem Curve

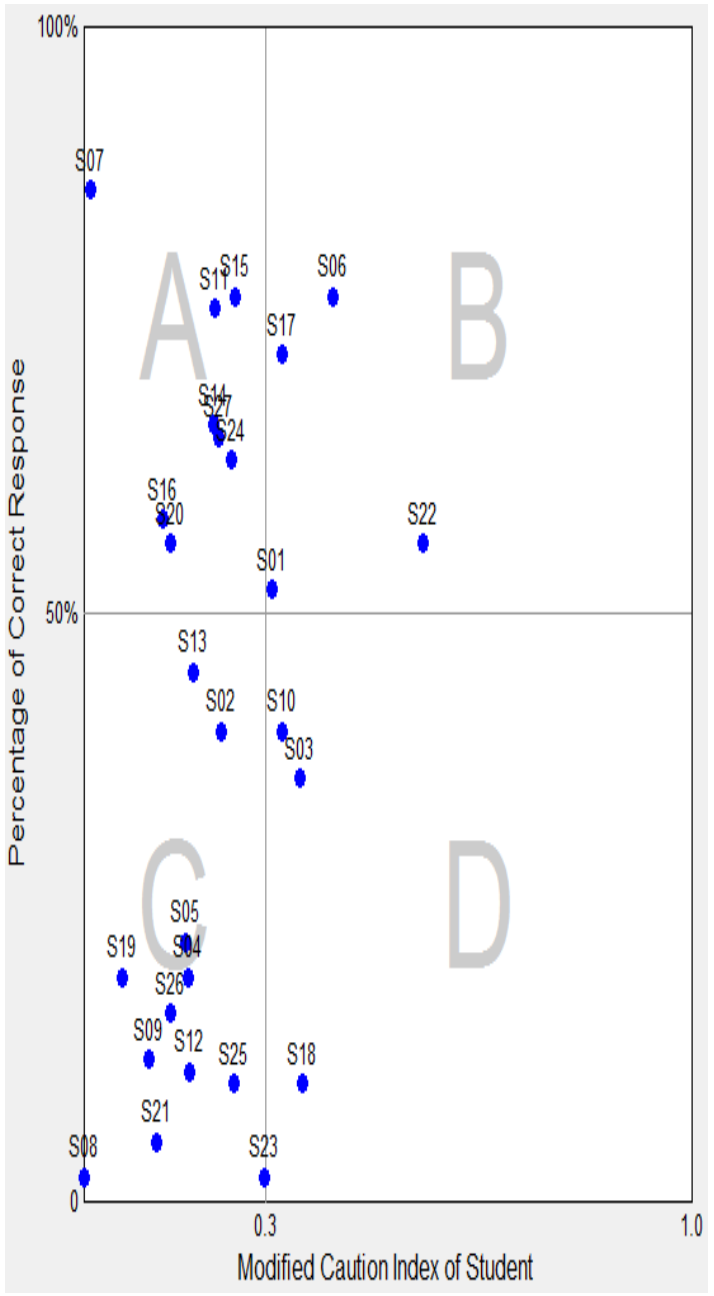

Figure 5. Student Diagnostic Graph students

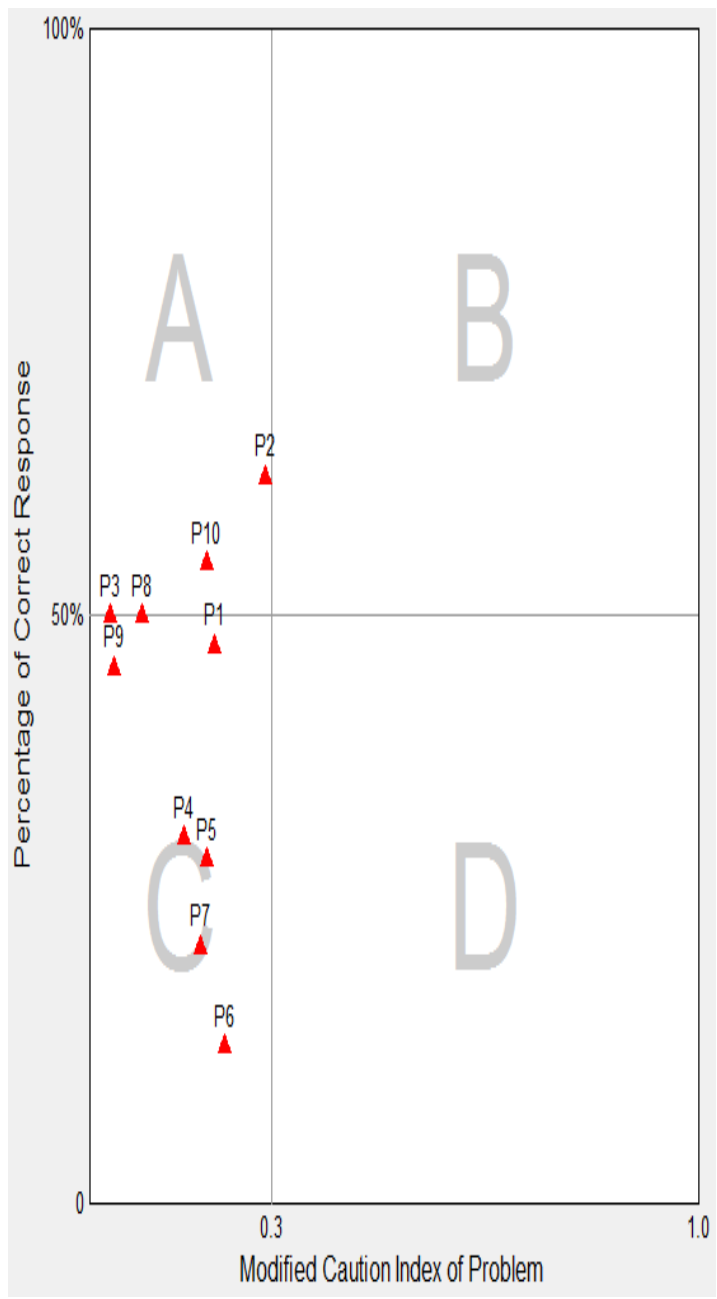

Figure 6. Problem Diagnostic Graph students 


\section{Conclusion}

S-P Chart Analysis is an effective method for diagnosing learning. However, it only handles dichotomous data. Therefore, it only evaluates the students who answer an item correctly without considering the students who only answer a part of the item correctly. This study proposed the Partial Credit S-P Chart Analysis for analyzing partial credit test. The proposed method has overcome the disadvantages of traditional S-P Chart Analysis. At the same time, the study has successfully developed a new learning diagnosis tool. The PCSP 1.0 software developed by this study analyzes partial credit test according to the Partial Credit S-P Chart Analysis. It has two main advantages. The first one is that it analyzes the test data with small sample. So this software is very suitable to use in analyzing the test results of students in small classes. The second one is that the test result is analyzed by the software according to the proposed method that considers the students who only answer a part of the item. Thus, this software can assist teachers in assessing students' partial understanding. In addition, PCSP 1.0 software is designed for ease of use and time-saving, but the output is accurate and clearly visual. Its functions are as follows:

1) PCSP 1.0 software calculates and provides the result of analyzing S-P chart in the numeral form. The provided results include Cronbach's alpha, total score of student, correct answer rate of student, the student's Modified Caution Index, student type, correct answer total of problem, correct answer rate of problem, the problem's Modified Caution Index and problem type.

2) PCSP 1.0 software presents the analysis result visually by tables and graphs. The presented results include S-P chart, the S-P curve graph, the diagnosis graph for students, the diagnosis graph for problems, the distribution graph of total score and the distribution graph of problem difficulty.

3) PCSP 1.0 software also handles dichotomous data according to traditional S-P Chart Analysis.

In short, PCSP 1.0 software is a useful tool for teachers in diagnosing the learning status of the student and assessing test item quality.

\section{References}

[1] T. Sato, "The SP chart and the caution index", NEC educational information bulletin, vol. 80, no. 1, (1980).

[2] T. W. Sheu, D. H. Pham, P. T. Nguyen, and P. H. Nguyen, "A Matlab Toolbox for StudentProblem Chart and Grey Student-Problem Chart and Its Application", International Journal of Kansei Information, vol. 4, no. 2, (2013).

[3] D. L. McArthur, "Analysis of Test Score Patterns: The Student - Problem (S - P) Technique", CSE Report, vol. 218, (1983).

[4] X. Wang, "Teachers' Views on Conducting Formative Assessment in Chinese Context", Engineering Letters, vol. 16, no. 2, (2008).

[5] M. N. Yu, Educational Testing and Assessment, Third ed. Taipei: Psychology Publisher (2011).

[6] C. H. Wang and C. P. Chen, "Employing Online S-P Diagnostic Table for Qualitative Comments on Test Results", The Electronic Journal of e-Learning, vol. 11, no. 3, (2013).

[7] C. P. Chuang, B. Y. Zheng, H. C. Chien, Y. J. Huang, and N. T. Hsieh, "Learning Effects of Blended Learning at Different Ratios With SP Chart", International Journal of Information Technology \& Computer Science, vol. 6, no. 2, (2012).

[8] D. W. S. Tai, R. C. Zhang, and J. L. Chen, "An Evaluation of the Visual Presentation eLearning on Engineering Drawing by Using the SP Chart Analysis", International Journal of eEducation, e-Business, e-Management and e-Learning, vol. 4, no. 3, (2014).

[9] D. H. Pham, T. W. Sheu, and M. Nagai, "Assessment Academic Achievement of Student in Physics with S-P chart", Journal of Science, vol. 24 (2013). 
[10] T. W. Sheu, D. H. Pham, C. P. Tsai, P. T. Nguyen, P. H. Nguyen, and M. Nagai, "Rasch GSP Toolbox for Assessing Academic Achievement", Journal of Software, vol. 9, no. 7, (2014).

[11] W. C. Chang, H. C. Yang, T. K. Shih, and M. F. Li, "Integrating IRT to estimate learning ability with SP chart in web based learning environment", in Advances in Blended Learning, Edited E. W. C. Leung, F. L. Wang, L. Miao, J. Zhao, and J. He, Springer-Verlag Berlin Heidelberg, Germany (2008), pp. 133-145.

[12] J. M. Yih, Y. H. Lin, and T. W. Sheu, "Integration of SP Chart and POT for Cognition Diagnosis and Application on Capacity Concepts Analysis", Proceedings of the 7th WSEAS International Conference on E-ACTIVITIES (E-ACTIVITIES '08), (2008) December 29-31; Cairo, Egypt.

[13] D. J. Chen, A. F. Lai, and I. C. Liu, "The design and implementation of a diagnostic test system based on the enhanced SP model", Journal of Information Science and Engineering, vol. 21, no. 5, (2005).

[14] D. L. Harnisch and R. L. Linn, "Analysis of item response patterns: Questionable test data and dissimilar curriculum practices", Journal of Educational Measurement, vol. 18, no. 3, (1981).

[15] T. MathWorks, MATLAB®Compiler ${ }^{\mathrm{TM}}$ User's Guide, (2014).

[16] T. MathWorks, MATLAB® Creating Graphical User Interfaces , (2012).

[17] H. Moore, MATLAB for Engineers, Third ed. New Jersey: Prentice Hall, (2012).

[18] G. Q. Chen and J. L. Kang, "Development of a MATLAB Toolbox for 3-PRS Parallel Robot," International Journal of Hybrid Information Technology, vol. 7, no. 5, (2014).

[19] M. Kurata and T. Sato, "Simulation and Analysis of the Item Score Table Using an S-P Score Mode", The Japanese Journal of Behaviormetrics, vol. 4, no. 1, (1976).

[20] T. Sato, An Introduction to the S-P chart. Tokyo: Meiji Tosho, (1996).

[21] M. M. C. Mok, S. M. Lam, M. Y. Ngan, J. J. Yao, M. Y. W. Wong, J. K. Xu, and S. Y. C. Ting, "Student-problem chart: An essential tool for SLOA", in Self-directed Learning Oriented Assessments in the Asia-Pacific, vol. 18, Edited M. M. C. Mok, Springer Netherlands, (2013), pp. 203-221.

\section{Authors}

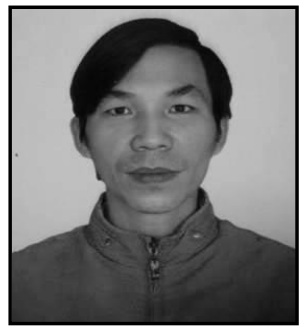

Duchieu Pham, he received the Master degree in Education at Hanoi Pedagogical University $\mathrm{N}^{\mathrm{0}} 2$, Vietnam in 1999 . He works as a lecturer of Primary Education Faculty of Hanoi Pedagogical University $\mathrm{N}^{0} 2$, Vietnam. He is also a Ph.D. student in Graduate Institute of Educational Information and Measurement, National Taichung University, Taiwan now. His research interests include grey system theory, educational measurement, and primary education.

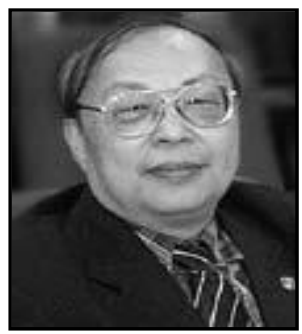

Tianwei Sheu, he received the Ph.D. degree in Mathematics from National Osaka University, Japan in 1990. He is a Professor of Graduate Institute of Educational Information and Measurement, National Taichung University, Taichung, Taiwan. $\mathrm{He}$ is also the director of TKIA (Taiwan Kansei Information Association). His studies focus on IRT, educational measurement, and E-learning. 


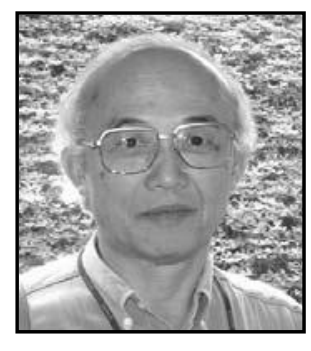

Masatake Nagai. He received Master's degree in Engineering from Toukai University, Japan in 1969. He worked in Oki Electric Industry Co., Ltd. for 18 years and was mainly engaged in the design development of ME systems, communication network systems, OA systems, etc. He was also a researcher (Dr. Matsuo research) at the Tohoku University while working toward his Ph.D in Engineering. From 1989, he worked at the Teikyo University Department of Science and Engineering as an assistant professor and eventually as an engineering professor. $\mathrm{He}$ is a Chair Professor in Graduate Institute of Educational Information and Measurement, National Taichung University, Taiwan now. His research interests include approximation, strategy system engineering, information communication network technology, agent, kansei information processing, grey system theory, and engineering application. 
International Journal of Hybrid Information Technology Vol.8, No.6 (2015) 